\title{
Outage Probabilities of Wireless Systems with LCMV Beamforming
}

\author{
Hanyu Li, Student Member, IEEE, Yu-Dong Yao, Senior Member, IEEE, and Jin Yu, Member, IEEE
}

\begin{abstract}
This paper investigates the outage probability of a wireless system with linear constrained minimum variance (LCMV) beamforming using a uniform linear array beamformer. LCMV beamforming is able to perfectly cancel a number of dominant interferers while other interferers remain. A simplified beamforming model is used to derive closed-form outage probability expressions considering the impact of LCMV beam patterns on various interferers. Fading statistics of Rayleigh, Rician, and Nakagami are used to characterize the desired signal, whereas interferers are assumed to be subject to Rayleigh fading. One important aspect of this paper is the consideration of the directions of arrivals (DOA) of the dominant interferers and the exact beam patterns in the outage performance evaluations of LCMV beamforming systems. Numerical results of the outage probability are presented to illustrate the impact of DOA's of the dominant interferers and the impact of different fading scenarios. The paper also presents performance comparison between LCMV beamforming and conventional beamforming considering different interference scenarios (DOA's of dominant interferers).
\end{abstract}

Index Terms - LCMV beamforming, Nakagami fading, outage probability, Rayleigh fading, Rician fading.

\section{INTRODUCTION}

A NTENNA array techniques are based on utilizing multiple antenna elements to achieve performance and capacity enhancement without the need for additional power or spectrum [1]. There are two categories of antenna array techniques which are diversity and beamforming. Diversity and beamforming differ in the spacing requirement among antenna elements. Diversity techniques use a number of antennas which are separated far apart from each other [2]. This approach was first developed for a receiver side and, lately, it has been used at a transmitter side. Transmit antenna diversity and receive antenna diversity can also be implemented at the same time. Space-time coding, a popular technique nowadays, is developed based on transmit diversity [3]. The second category of antenna array techniques is beamforming. This technique uses several antenna elements which are placed very close to each other to form an antenna beam [4], [5]. The beam can be steered and focus most signal energy toward a desired direction. In the same time it reduces interference

Manuscript received March 29, 2006; revised August 8, 2006; accepted September 28, 2006. The associate editor coordinating the review of this letter and approving it for publication was R. Mallik.

H. Li and Y.-D. Yao are with the Wireless Information Systems Engineering Laboratory (WISELAB), Department of Electrical and Computer Engineering, Stevens Institute of Technology, Hoboken, NJ 07030 USA (e-mail: \{hli5, yyao\}@stevens.edu).

J. Yu was with the Wireless Information Systems Engineering Laboratory (WISELAB), Department of Electrical and Computer Engineering, Stevens Institute of Technology, Hoboken, NJ, 07030 USA. He is now with Berkeley Varitronics Systems, Inc., Metuchen, NJ 08840 USA (e-mail: jyu@bvsystems.com).

Digital Object Identifier 10.1109/TWC.2007.060128. in other directions. Many research results for future wireless systems using antenna arrays have been reported [1]-[3], [5][8]. In this paper we focus on the beamforming techniques while evaluating wireless system outage probabilities.

Beamforming can be implemented through conventional beamforming, minimum variance distortionless response (MVDR) beamforming, or linear constrained minimum variance (LCMV) beamforming [4]. The MVDR beamforming is also known as optimum beamforming. The LCMV beamforming is developed from MVDR beamforming with additional linear constraints to improve its robustness [4]. In implementing conventional beamforming, the antenna mainlobe is steered toward a desired signal. In MVDR beamforming, an antenna pattern is formed to maximize the output signal-tointerference-plus-noise ratio while maintaining a constant gain in the direction of the desired signal [9]. The MVDR beamforming is sensitive to direction-of-arrival (DOA) estimation errors and its performance decreases significantly when an interferer is inside the mainlobe [4]. The LCMV beamforming can be implemented by placing nulls in the directions of interferers when multiple interferers are considered [4], [10]-[12]. To cancel/null all the interferers using LCMV beamforming, the number of antenna elements is required to exceed the number of nulls by one [5]. In a practical beamforming system the number of interferers can be larger than the number of antenna elements and there will be some residual interferers in the wireless system [5]. In this paper we study the performance of a wireless system that deploys a LCMV beamformer with the number of interferers more than the number of antenna elements.

The outage probability is an important performance measure for wireless systems [13], [14]. Performance in terms of the outage probability has been investigated for systems using antenna diversity techniques and, in [15], [16], and [17], several diversity combining schemes and various fading scenarios are considered. A generalized moment-generation-function (MGF) method was proposed to calculate the outage probability for diversity systems in [18]. The outage probability of wireless systems with beamforming has also been investigated [10]-[12], [19]. Conventional beamforming is examined in [19] and outage probability expressions for Rayleigh, Rician, or Nakagami faded signal and Rayleigh faded interferers are derived. LCMV beamforming scenarios are studied in [10][12]. The LCMV beamformer perfectly cancels $L$ strongest interferers while all other interferers remain, where $L$ is determined by the number of antenna elements. In the outage performance evaluations presented in [10]-[12], the effect of the LCMV beamforming is characterized by reducing the total number of interferers by $L$ ( $L$ strongest interferers). It is noted that the LCMV beam pattern impact on the 


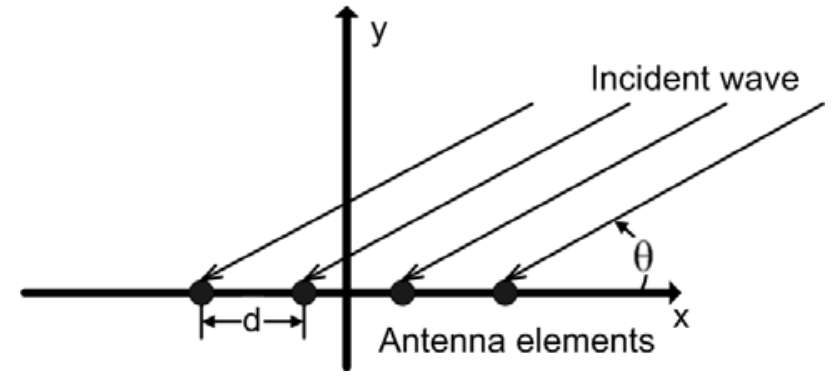

Fig. 1. A uniform linear array (ULA).

remaining interferers is ignored. A full characterization of the LCMV beamforming impact should have two basic elements, (1) the elimination of $L$ strongest interferers and (2) power level scaling (attenuation or increase) of the remaining interferers depending on the beam pattern. However, outage performance evaluations with those considerations have not been conducted. This paper differs from previous research, including [10]-[12], in several aspects. First, the beam pattern of a LCMV beamformer is considered in the derivation of the outage probability expressions. Second, the LCMV beam pattern is determined considering the directions of $L$ strongest interferers. Third, the residual interferers are assumed to be uniformly distributed in all directions and a simplified beamforming model is used to quantify the interference impact. In the following sections, closed-form outage probabilities will be derived for scenarios with a Rayleigh, Rician, or Nakagami faded signal and Rayleigh faded interferers.

The remainder of this paper is organized as follows. The system model is given in Section II. Outage probability expressions are derived in Section III. A simplified beamforming model is also introduced in this section. Numerical results are given in Section IV and, finally, conclusions are drawn in Section V.

\section{SySTEM MODEL}

\section{A. Beamforming}

While few antenna elements could be implemented at a mobile station, large antenna arrays can be installed at a base station. At a base station, receive beamforming for each desired signal can be implemented independently without affecting the performance of other links [20]-[22]. A uniform linear array (ULA) in a two-dimensional environment is considered and shown in Fig. 1. The distance $d$ between the antenna elements is assumed to be $0.5 \lambda$, where $\lambda$ is the carrier wavelength, and $\theta$ is an arrival angle of incident waves. In the ULA system, a combining network connects an array of low gain antenna elements and generates an antenna pattern [4],

$$
G=\left|\mathbf{W}^{H} \mathbf{V}(\psi)\right|^{2}
$$

where $\psi$ is a scan angle, $\mathbf{W}^{H}$, a $1 \times M$ vector, is a weight vector for antenna elements, $M$ is the number of antenna elements, and $\mathbf{V}(\psi)$, an $M \times 1$ vector, is an array manifold vector

$$
\begin{aligned}
& \mathbf{V}(\psi)=\left[e^{-j\left(\frac{M-1}{2}\right) \pi \cos (\psi)} \quad e^{-j\left(\frac{M-1}{2}-1\right) \pi \cos (\psi)}\right. \\
& \left.\ldots \quad e^{j\left(\frac{M-1}{2}\right) \pi \cos (\psi)}\right]^{T}
\end{aligned}
$$

The beam could be steered to a desired direction by varying $\psi$. For conventional beamforming, the weight factor is

$$
\mathbf{W}_{c}=\frac{1}{M} \mathbf{V}(\theta)
$$

and the beam pattern can be simplified as [21], [23],

$$
G(\psi, \theta)=\left|\frac{\sin (0.5 M \pi(\cos \theta-\cos \psi))}{M \sin (0.5 \pi(\cos \theta-\cos \psi))}\right|^{2}
$$

If LCMV beamforming is considered, the weight factor is expressed as [4],

$$
\mathbf{W}_{L C M V}^{H}=\mathbf{g}^{H}\left[\mathbf{C}^{H} \mathbf{S}_{n}^{-1} \mathbf{C}\right]^{-1} \mathbf{C}^{H} \mathbf{S}_{n}^{-1}
$$

where $\mathbf{S}_{n}$ is a noise spectral matrix which characterize both noise and interference, $\mathbf{C}$ is an $M \times M_{c}$ matrix, and $\mathbf{g}^{H}$ is a $1 \times M_{c}$ vector. Considering $M_{c}$ linear constraints for LCMV beamforming, the constraint equation is

$$
\mathbf{W}_{L C M V}^{H} \mathbf{C}=\mathbf{g}^{H}
$$

The LCMV beamforming in [10]-[12] assumes null constraints. When the number of interferers is larger than the number of antenna elements, the maximum number of nulls that the LCMV beamformer can generate is $M-1$, and the number of constraints $M_{c}=M$. Thus we have

$$
\mathbf{C}_{L C M V}=\left[\begin{array}{lllll}
\mathbf{V}(\theta) & \mathbf{V}\left(\theta_{1}\right) & \mathbf{V}\left(\theta_{2}\right) & \ldots & \mathbf{V}\left(\theta_{M-1}\right)
\end{array}\right]
$$

and

$$
\mathbf{g}_{L C M V}^{H}=\left[\begin{array}{lllll}
1 & 0 & 0 & \ldots & 0
\end{array}\right]
$$

where $\theta_{i}$ is the arrival angle of interferer $i$. Notice that the antenna gain toward the desired signal has been normalized to one. Insert (7) and (8) into (5) and the LCMV beam pattern can be written as

$$
G\left(\psi, \theta, \theta_{1}, \theta_{2}, \ldots, \theta_{M-1}\right)=\left|\mathbf{g}_{L C M V}^{H} \mathbf{C}_{L C M V}^{-1} \mathbf{V}(\psi)\right|^{2}
$$

In the remaining of this paper, we will use the antenna pattern specified in (9) to evaluate the outage probability of systems with LCMV beamforming.

\section{B. Fading Model}

The fading statistics of the desired signal and interferers can be different[24], [25]. In this paper we assume the desired signal is subject to Rayleigh, Rician, or Nakagami fading, whereas interferers are assumed to be independently Rayleigh faded with the same mean power. Rayleigh fading is commonly used to describe the wireless communication channel. The probability density function (pdf) of Rayleigh faded signal power, $x$, is

$$
p_{x}(x)=\frac{1}{X} \exp \left(-\frac{x}{X}\right), x \geqslant 0
$$

where $X$ is the mean power. When there is a line-of-sight between the desired signal and the base station, the desired signal follows a Rician distribution, and its power, $x$, follows the pdf

$$
\begin{aligned}
p_{x}(x) & =\frac{\left(1+K_{d}\right) e^{-K_{d}}}{X} \exp \left(-\frac{\left(1+K_{d}\right) x}{X}\right) \\
& \times \mathrm{I}_{0}\left(2 \sqrt{\frac{K_{d}\left(1+K_{d}\right) x}{X}}\right)
\end{aligned}
$$


where $K_{d}$ is a Rician factor which ranges from 0 to $\infty$ and $\mathrm{I}_{0}(\cdot)$ is the zero-th order modified Bessel function of the first kind. The Rician fading degenerates to Rayleigh fading when $K_{d}=0$. Another suitable statistics to characterize a wireless channel is Nakagami fading,

$$
p_{x}(x)=\frac{m_{d}^{m_{d}} x^{m_{d}-1}}{X^{m_{d}} \Gamma\left(m_{d}\right)} \exp \left(-\frac{m_{d}}{X} x\right)
$$

where $\Gamma(\cdot)$ is the Gamma function and $m_{d}$ is a parameter of Nakagami fading. $1 / m_{d}$ is the amount of fading which is defined as the ratio of the variance to the square of the mean received energy. By setting $m_{d}=1$, the Nakagami fading (12) reduces to Rayleigh fading.

There are $N_{I}$ interferers, which are assumed to follow identical independent Rayleigh fading. The pdf of interferer $i$ 's power, $y_{i}$, is $p_{y_{i}}\left(y_{i}\right)=\frac{1}{Y} \exp \left(-\frac{y_{i}}{Y}\right), y_{i} \geqslant 0$, where $Y$ is the mean power. The LCMV beamforming is able to cancel out $L(L=M-1)$ strongest interferers. We need to calculate the pdf of the residual interferers. As there are $N_{I}$ interferers with power levels, $y_{1}, y_{2}, \ldots, y_{N_{I}}$, we sort the interferers according to their power levels and obtain $y_{\left(N_{I}\right)} \geq y_{\left(N_{I}-1\right)} \geq \ldots \geq$ $y_{(2)} \geq y_{(1)}>0$. After nulling $L$ strongest interferers, we have $N\left(N=N_{I}-L\right)$ remaining interferers and their joint pdf is given by [10]

$$
\begin{aligned}
& p_{y_{(1)}, y_{(2)}, \ldots, y_{(N)}}\left(y_{(1)}, y_{(2)}, \ldots, y_{(N)}\right)=\frac{N_{I} !}{\left(N_{I}-N\right) ! Y^{N}} \\
& \times \exp \left[-\frac{1}{Y}\left(\sum_{n=1}^{N} y_{(n)}+\left(N_{I}-N\right) y_{(N)}\right)\right]
\end{aligned}
$$

\section{Outage Probability}

In interference limited wireless systems, adequate signal-tointerference ratio (SIR) is essential for successful communications [24], [25]. Therefore, the outage probability, defined as the probability of failing to achieve a SIR sufficiently large to achieve satisfactory reception, is an appropriate measure to characterize the performance of the wireless system. Mathematically the outage probability, $P_{\text {out }}$, is defined as $P_{\text {out }}=\int_{0}^{\gamma_{t h}} p_{\gamma}(\gamma) d \gamma$, in which $\gamma$ is an instantaneous SIR, $\gamma_{t h}$ is a required threshold. $\gamma$ is related to $x$ and $y$, where $x$ is the power of a desired signal and $y$ is the total interference power. In this paper, we consider that the desired signal follows Rayleigh, Rician, or Nakagami fading, and each interferer follows a Rayleigh distribution.

There are other performance criterions such as bit error rates and symbol error rates to quantify wireless system performance. These performance measures and results are related to specific modulation schemes and transceiver structures. Therefore in this paper we focus on the outage probability evaluation for beamforming systems.

\section{Derivation of Outage Probability}

In this section, the outage probability will be derived. We will start with deriving a general outage probability expression using the exact LCMV beam patterns for both signal and interferers. We will then introduce a simplified beamforming model, which is used to derive closed-form outage probability expressions.

\section{A. Outage Probability Evaluation Using Exact LCMV Beam Pattern}

A LCMV beamformer with $M$ antenna elements can put nulls in the directions of the $M-1(L)$ strongest interferers. The received power from a residual interferer is scaled (attenuated or increased) by a factor determined by its direction, which is $G\left(\psi_{i}, \theta, \theta_{1}, \theta_{2}, \ldots, \theta_{M-1}\right)$ as in (9), where $\psi_{i}$ is the DOA of $i$ th residual interferer. The instantaneous total power of residual interferers is then

$$
y=\sum_{i=1}^{N} G\left(\psi_{i}, \theta, \theta_{1}, \theta_{2}, \ldots, \theta_{M-1}\right) y_{(i)}
$$

In previous sections the pdf of signal power $x$ has been given by (10)-(12) and the joint pdf of residual interference power has been given in (13). The SIR, $\gamma$, in LCMV beamforming is expressed as

$$
\gamma=\frac{x}{\sum_{i=1}^{N} G\left(\psi_{i}, \theta, \theta_{1}, \theta_{2}, \ldots, \theta_{M-1}\right) y_{(i)}}
$$

and the conditional outage probability for given directions of residual interferers is

$$
\begin{aligned}
& P_{\text {out }}\left(\psi_{1}, \psi_{2}, \ldots, \psi_{N}\right) \\
& =\operatorname{Pr}\left[\frac{x}{\sum_{i=1}^{N} G\left(\psi_{i}, \theta, \theta_{1}, \theta_{2}, \ldots, \theta_{M-1}\right) y_{(i)}}<\gamma_{t h}\right] \\
& =\int_{0}^{\gamma_{\text {th }}} \int_{0}^{\infty} y p_{x}(\gamma y) \frac{d}{d y}\left\{\int_{0}^{y} \int_{0}^{\hat{y}_{N-1}} \ldots \int_{0}^{\hat{y}_{1}}\right. \\
& \frac{N_{I} !}{\left(N_{I}-N\right) ! Y^{N}} \exp \left[-\frac{1}{Y}\left(\sum_{n=1}^{N} y_{(n)}+\left(N_{I}-N\right) y_{(N)}\right)\right] \\
& d y_{\left.(1) \ldots d y_{(N-1)} d y_{(N)}\right\} d y d \gamma}
\end{aligned}
$$

where $\hat{y}_{j}=\min \left[\left(y-\sum_{i=j+1}^{N} G\left(\psi_{i}\right) y_{(i)}\right), y_{(j+1)}\right]$. As shown in Appendix A, we are able to derive and obtain expressions for the conditional outage probability. If the signal is subject to Rayleigh fading, following (A-20) and [24], [25], we have

$$
P_{\text {out }}\left(\psi_{1}, \psi_{2}, \ldots, \psi_{N}\right)=\sum_{i=1}^{N} A_{i}\left[1-\left(1+\frac{1}{S I R \times C_{i}}\right)^{-1}\right]
$$

in which

$$
A_{i}=\prod_{j=1, j \neq i}^{N}\left(1-\frac{C_{i}}{C_{j}}\right)^{-1}
$$

and

$$
C_{i}=\frac{N_{I}-i+1}{\sum_{k=i}^{N} G\left(\psi_{k}, \theta, \theta_{1}, \theta_{2}, \ldots, \theta_{M-1}\right)}
$$

and $S I R$ is a normalized signal to interference ratio, and $S I R=\frac{X}{Y \gamma_{t h}}$.

If the signal is subject to Rician fading,

$$
\begin{gathered}
P_{\text {out }}\left(\psi_{1}, \psi_{2}, \ldots, \psi_{N}\right)=\sum_{i=1}^{N} A_{i} \exp \left(-\frac{K_{d}}{1+\frac{K_{d}+1}{S I R \times C_{i}}}\right) \\
\times\left(1+\frac{S I R \times C_{i}}{K_{d}+1}\right)^{-1}
\end{gathered}
$$


For Nakagami faded signal,

$$
\begin{gathered}
P_{\text {out }}\left(\psi_{1}, \psi_{2}, \ldots, \psi_{N}\right)=\sum_{i=1}^{N} A_{i} \frac{\Gamma\left(m_{d}+1\right)}{m_{d} \Gamma\left(m_{d}\right)}\left(\frac{m_{d}}{S I R \times C_{i}}\right)^{-m_{d}} \\
\quad \times{ }_{2} \mathrm{~F}_{1}\left(m_{d}+1, m_{d}, 1+m_{d},-\frac{m_{d}}{S I R \times C_{i}}\right)
\end{gathered}
$$

where ${ }_{2} \mathrm{~F}_{1}(., ., .,$.$) is the Gauss hypergeometric function de-$ fined in [26],

$$
{ }_{2} \mathrm{~F}_{1}(a, b, c, z)=\frac{\Gamma(c)}{\Gamma(a) \Gamma(b)} \sum_{n=0}^{\infty} \frac{\Gamma(a+n) \Gamma(b+n)}{\Gamma(c+n)} \cdot \frac{z^{n}}{n !}
$$

Considering that the residual interferers are uniformly distributed in all directions, $[0,2 \pi)$, the average outage probability can be expressed through multiple integrals

$$
P_{\text {out }}=\int_{0}^{2 \pi} \ldots \int_{0}^{2 \pi} \frac{1}{(2 \pi)^{N}} P_{\text {out }}\left(\psi_{1}, \psi_{2}, \ldots, \psi_{N}\right) d \psi_{1} \ldots d \psi_{N}
$$

in which $P_{\text {out }}\left(\psi_{1}, \psi_{2}, \ldots, \psi_{N}\right)$ is given in (17), (20), and (21) for different fading signal scenarios. Notice that (23) gives the exact outage probability expression as it takes full considerations of LCMV beam pattern $\left(G\left(\psi_{k}, \theta, \theta_{1}, \theta_{2}, \ldots, \theta_{M-1}\right)\right.$, $k=1,2, \ldots, N)$. Numerical evaluations of (23) is possible when $N$ is small and results for cases with $N=1,2$, and 3 are presented in Section IV. However, for larger $N$, the evaluation of (23) becomes cumbersome or infeasible. In the following section a simplified beamforming model will be introduced and used to derive closed-form outage probability solutions. The accuracy of the simplified model will be addressed in Section IV.

\section{B. Introduction of Simplified and Accurate Beamforming Model}

The complexity in considering the exact beam pattern can be high, especially for performance evaluation under multiple residual interferers due to multiple integrals. A simple Bernoulli model is studied in [27] in which a signal is considered to be either within a mainlobe $(G=1)$ or out of the mainlobe $(G=0)$ and the half-power beamwidth is defined as the beamwidth. This model is easy to use, but it neglects the impact of sidelobes and the effect of any specific beam patterns. Reference [28] provides a beamforming model with a triangular pattern to characterize the mainlobe of a beam. In [29], an accurate, yet simple, beamforming model is developed to account the impact of sidelobes and the exact beam patterns. In dealing with interference, this model simplifies the beam pattern to a flat mainlobe and a flat sidelobe. There are two parameters, mainlobe width $B$ (normalized by $2 \pi$ ) and the sidelobe gain $\alpha$, associated with the model. The two parameters are determined based on a given beam pattern as

$$
\alpha=\frac{\mathrm{E}\left[G^{2}\left(\psi, \theta, \theta_{1}, \ldots, \theta_{M-1}\right)\right]-\mathrm{E}\left[G\left(\psi, \theta, \theta_{1}, \ldots, \theta_{M-1}\right)\right]}{\mathrm{E}\left[G\left(\psi, \theta, \theta_{1}, \ldots, \theta_{M-1}\right)\right]-1}
$$

and

$$
B=\frac{\left.\mathrm{E}\left[G^{2}\left(\psi, \theta, \theta_{1}, \ldots, \theta_{M-1}\right)\right]-\mathrm{E}^{2}\left[G\left(\psi, \theta, \theta_{1}, \ldots, \theta_{M-1}\right)\right]\right)}{\mathrm{E}\left[G^{2}\left(\psi, \theta, \theta_{1}, \ldots, \theta_{M-1}\right)\right]+1-2 \mathrm{E}\left[G\left(\psi, \theta, \theta_{1}, \ldots, \theta_{M-1}\right)\right]}
$$

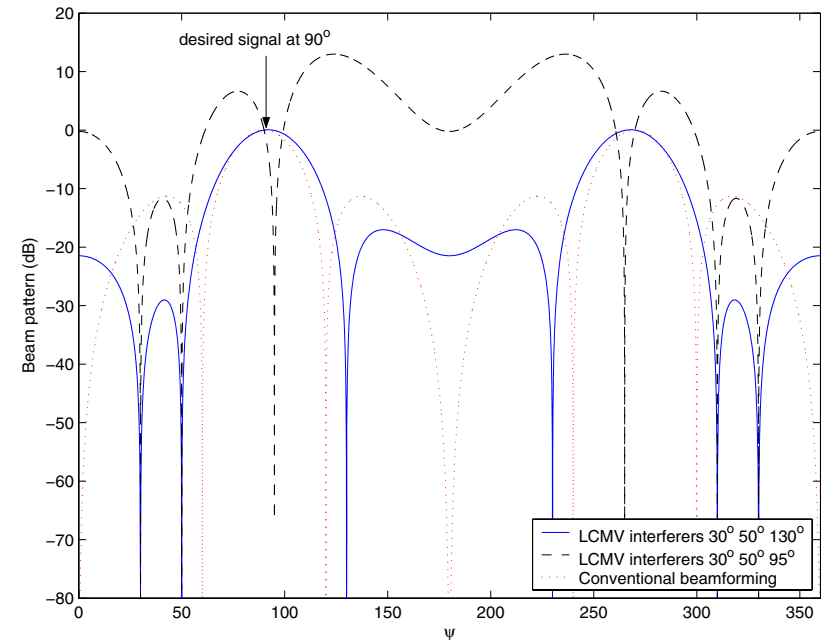

(a)

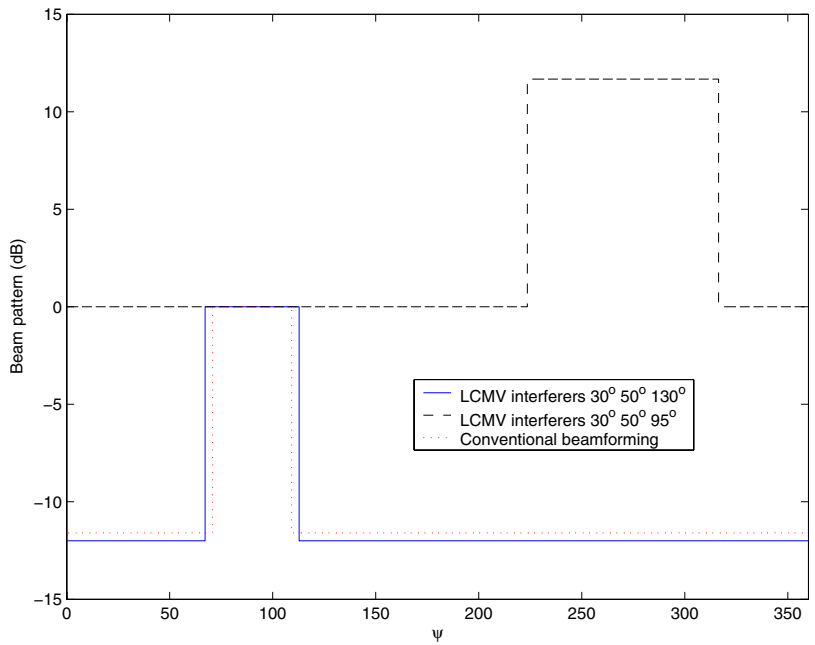

(b)

Fig. 2. A simplified model for beamforming, desired signal arrival angle $\theta=90^{\circ}$ (a) Signal model, (b) Interference model.

where $\mathrm{E}\left[G\left(\psi, \theta, \theta_{1}, \ldots, \theta_{M-1}\right)\right]$ and $\mathrm{E}\left[G^{2}\left(\psi, \theta, \theta_{1}, \ldots, \theta_{M-1}\right)\right]$ are the antenna gain and square of the antenna gain averaged with respect to random variables, $\psi, \theta, \theta_{1}, \ldots$, and $\theta_{M-1}$. It is important to point out that, in this simplified beamforming model, two beam patterns are considered and utilized. One is an exact beam pattern which is used to evaluate a desired signal (Fig. 2 (a)) and the other is a simplified beam pattern for interferers (Fig. 2 (b)). A conventional beamforming is also plotted in Fig. 2 for comparison. More detailed discussions of the beam patterns (Fig. 2) will be given in Section IV.

\section{Outage Probability Evaluation Using the Simplified Beam- forming Model}

In this section, the outage probability of a wireless system using LCMV beamforming will be derived, and closed-form expressions are given utilizing the simplified beamforming model. The residual interferers are assumed to be uniformly distributed in the directions of arrivals $[0,2 \pi)$. Considering the simplified beamforming model, the probability that the $i$ th residual interferer falls within the beam mainlobe is $B$ 
and interference power is $y_{(i)}$, while the probability that the interferer is in the sidelobe is $1-B$ and its interference power is $\alpha \times y_{(i)}$. Let $l_{i}$ be a Bernoulli random variable which indicates whether the $i$ th residual interferer is in the mainlobe or sidelobe.

$\left\{\begin{array}{l}\operatorname{Pr}\left[l_{i}=1\right]=B, i \text { th residual interferer is in mainlobe } \\ \operatorname{Pr}\left[l_{i}=0\right]=1-B, i \text { th residual interferer is in sidelobe }\end{array}\right.$

Now the conditional total interference power is

$$
y=\sum_{i=1}^{N}\left(l_{i} y_{(i)}+\left(1-l_{i}\right) \alpha y_{(i)}\right)
$$

The SIR, $\gamma$, using the simplified beamforming model, is expressed as

$$
\gamma=\frac{x}{\sum_{i=1}^{N}\left(l_{i} y_{(i)}+\left(1-l_{i}\right) \alpha y_{(i)}\right)}
$$

The pdf of signal power $x$ is given is (10)-(12) and the joint pdf of $y_{(i)}$ is given in (13). The conditional outage probability is

$$
\begin{aligned}
& P_{\text {out }}\left(l_{1}, l_{2}, \ldots, l_{N}\right) \\
& =\operatorname{Pr}\left[\frac{x}{\sum_{i=1}^{N}\left(l_{i} y_{(i)}+\left(1-l_{i}\right) \alpha y_{(i)}\right)}<\gamma_{t h}\right] \\
& =\int_{0}^{\gamma_{t h}} \int_{0}^{\infty} y p_{x}(\gamma y) \frac{d}{d y}\left\{\int_{0}^{y} \int_{0}^{\ddot{y}_{N-1}} \cdots \int_{0}^{\ddot{y}_{1}}\right. \\
& \frac{N_{I} !}{\left(N_{I}-N\right) ! Y^{N}} \exp \left[-\frac{1}{Y}\left(\sum_{n=1}^{N} y_{(n)}+\left(N_{I}-N\right) y_{(N)}\right)\right] \\
& \left.d y_{(1)} \ldots d y_{(N-1)} d y_{(N)}\right\} d y d \gamma
\end{aligned}
$$

where $\ddot{y}_{j}=\min \left[\left(y-\left(l_{j+1} y_{(j+1)}+(1-\right.\right.\right.$ $\left.\left.\left.\left.l_{j+1}\right) \alpha y_{(j+1)}\right)\right), y_{(j+1)}\right]$. As shown in Appendix B, (29) can be simplified considering various fading scenarios. If the signal is subject to Rayleigh fading, following (B-8) and [25], we are able to obtain

$$
\begin{aligned}
P_{\text {out }}\left(l_{1}, l_{2}, \ldots, l_{N}\right) & =\sum_{j=1}^{J} \sum_{i=1}^{N_{j}} A_{j i} \\
& \times\left(1-\left(1+\frac{1}{S I R \times D_{(j)}}\right)^{-i}\right)
\end{aligned}
$$

where $J, N_{j}, A_{j i}$, and $D_{(j)}$ are given in Appendix B.

If the desired signal is subject to Rician fading, following (B-8) and [25], the outage probability is

$$
\begin{aligned}
& P_{\text {out }}\left(l_{1}, l_{2}, \ldots, l_{N}\right)=\sum_{j=1}^{J} \sum_{i=1}^{N_{j}} A_{j i} \exp \left(-\frac{K_{d}}{1+\frac{K_{d}+1}{S I R \times D_{(j)}}}\right) \\
& \times\left(1+\frac{S I R \times D_{(j)}}{K_{d}+1}\right)^{-1}\left(\sum_{n=0}^{i-1}\left(1+\frac{\left(K_{d}+1\right)}{S I R \times D_{(j)}}\right)^{-n}\right. \\
& \left.\quad \times \sum_{j=0}^{n}\left(\begin{array}{c}
n \\
n-j
\end{array}\right) \frac{1}{j !}\left(\frac{K_{d}}{1+\frac{S I R \times D_{(j)}}{\left(K_{d}+1\right)}}\right)^{n}\right)
\end{aligned}
$$

For a Nakagami faded signal, following (B-8) and [24], the outage probability is

$$
\begin{aligned}
& P_{\text {out }}\left(l_{1}, l_{2}, \ldots, l_{N}\right)=\sum_{j=1}^{J} \sum_{i=1}^{N_{j}} \frac{A_{j i} \Gamma\left(m_{d}+i\right)}{m_{d} \Gamma(i) \Gamma\left(m_{d}\right)}\left(\frac{m_{d}}{S I R \cdot D_{(j)}}\right)^{-m_{d}} \\
& \quad \times{ }_{2} \mathrm{~F}_{1}\left(m_{d}+i, m_{d}, 1+m_{d},-\frac{m_{d}}{S I R \times D_{(j)}}\right)
\end{aligned}
$$

Considering that $l_{i}$ is a Bernoulli random variable with parameter $B$, the average outage probability can be finally expressed as

$$
P_{\text {out }}=\sum_{l_{1}=0}^{1} \ldots \sum_{l_{N}=0}^{1}\left(\prod_{i=1}^{N} B^{l_{i}}(1-B)^{1-l_{i}}\right) P_{\text {out }}\left(l_{1}, l_{2}, \ldots, l_{N}\right)
$$

where $P_{\text {out }}\left(l_{1}, l_{2}, \ldots, l_{N}\right)$ is given in (30), (31), and (32). Notice that the above average outage probability result obtained based on the simplified beamforming model is a closed-form expression (for easy numerical computation), as opposed to the multiple integral expression, (23), without the use of the simplified model.

\section{NuMERICAL RESULTS}

The exact beam patterns of the beamformers with a desired signal at the broadside direction $\left(\theta=90^{\circ}\right)$ are plotted in Fig. 2 (a). Two LCMV beamforming scenarios and one conventional beamforming scenario using 4 antenna-element beamformers are compared. All of the three beam patterns maintain a constant gain of $0 \mathrm{~dB}$ in the direction of the desired signal. The conventional beamforming steers the mainlobe toward the desired signal and mitigates each interferer by a factor (antenna gain) which depends on its direction. For LCMV beamforming, we first consider a scenario with three interferers at $30^{\circ}, 50^{\circ}$, and $130^{\circ}$. While three nulls are placed toward those directions, all other interferers, if any, are subject to various attenuations depending on their directions. Another LCMV beamforming scenario considered includes three interferers at $30^{\circ}, 50^{\circ}$, and $95^{\circ}$. Due to the fact that one of the interferers $\left(95^{\circ}\right)$ is very close to the desired signal $\left(90^{\circ}\right)$, the obtained beam pattern has an undesired feature of a high-gain sidelobe. This phenomenon is also observed in [4]. Although three nulls are generated $\left(30^{\circ}, 50^{\circ}\right.$, and $\left.95^{\circ}\right)$, any other potential interferers could degrade the system performance significantly due to increased received interference power. The simplified beamforming models corresponding to the exact beam patterns are plotted in Fig. 2 (b). For the first LCMV beamforming scenario $\left(30^{\circ}, 50^{\circ}\right.$, and $\left.130^{\circ}\right)$, we obtain a beam pattern with a narrow mainlobe and a low sidelobe. In the second LCMV beamforming scenario $\left(30^{\circ}, 50^{\circ}\right.$, and $\left.95^{\circ}\right)$, one of interferers is very close to the desired signal and the obtained beam pattern results in a very wide mainlobe and a very high sidelobe.

The accuracy of the simplified beamforming model is examined with results shown in Fig. 3. The outage probabilities using the exact beam pattern are given by (23) (multiple integrals) and those using the simplified beamforming model are given by (33) (closed-form). Both desired signal and interferers are assumed to be subject to Rayleigh fading while the desired signal is at $90^{\circ}$ and three dominant interferers are at $30^{\circ}, 50^{\circ}$, and $130^{\circ}$. It is seen that the evaluation results 


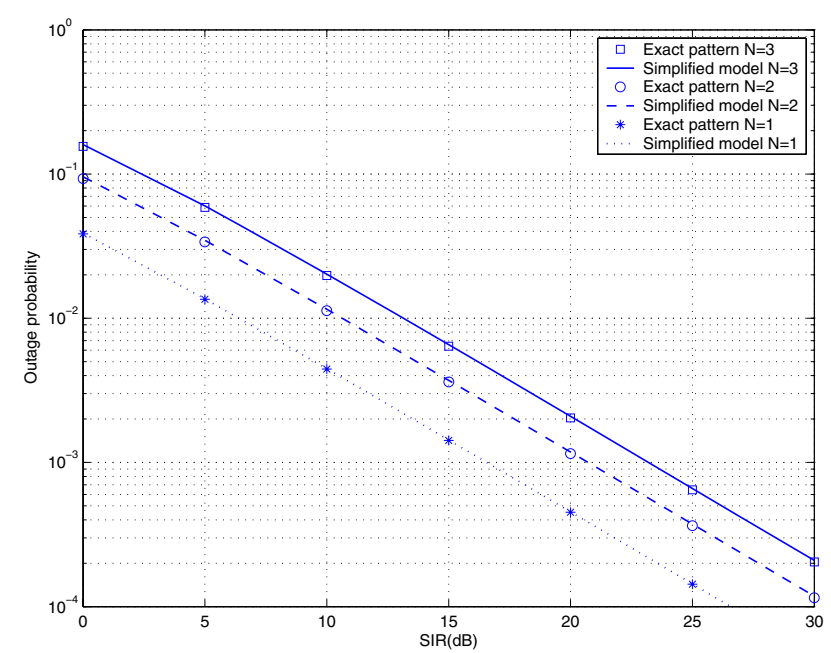

Fig. 3. Accuracy of the simplified beamforming model, $M=4$. Desired signal: Rayleigh fading and at $90^{\circ}$, interferers are Rayleigh faded and three dominant interferers are at $30^{\circ}, 50^{\circ}, 130^{\circ}$.

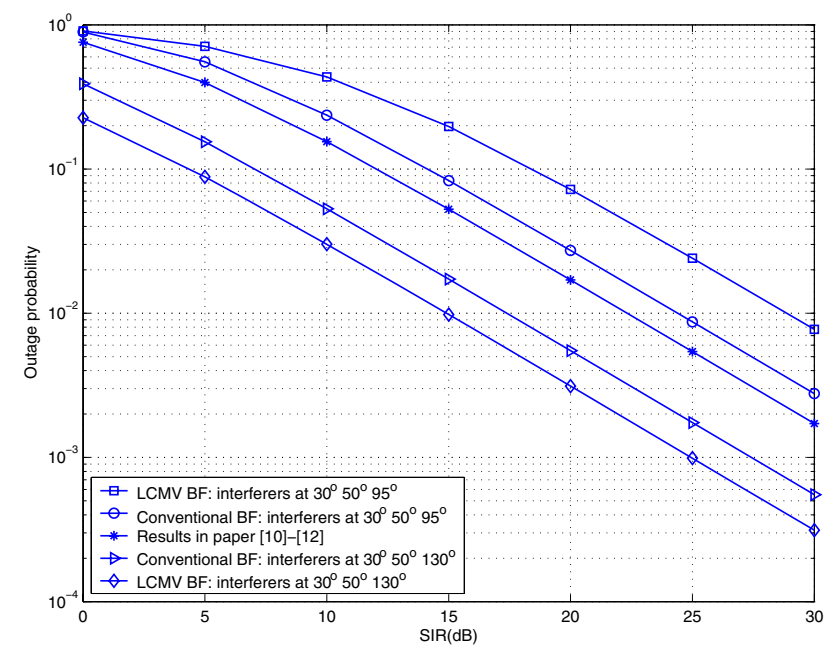

Fig. 4. Comparison of outage probabilities, $N_{I}=7, N=4$, and $M=4$. Desired signal: Rayleigh fading and at $90^{\circ}$, interferers: Rayleigh fading.

using the simplified model match those using the exact beam pattern very well. The scenarios with the number of residual interferers ranging from 1 to 3 are considered in Fig. 3. Results for larger number of residual interferers are not obtained due to the computational complexity of multiple integrals if the exact beam pattern is used (Eq. (23)). This also indicates the need to use the simplified beamforming model.

The outage probabilities with respect to different beamforming schemes are plotted in Fig. 4. LCMV beamforming results evaluated using the simplified beamforming model (Eq. (33)), conventional beamforming results [19], and LCMV beamforming results presented in [10]-[12] are compared. The number of antenna elements is 4 and the number of total interferers is assumed to be 7. For LCMV beamforming schemes, 3 strongest interferers are nulled and there are 4 residual interferers remaining. Two interference scenarios with the directions of the three strongest interferers at $\left(30^{\circ}, 50^{\circ}\right.$, $\left.130^{\circ}\right)$ and $\left(30^{\circ}, 50^{\circ}, 95^{\circ}\right)$ are considered. The desired signal is at $90^{\circ}$ and the corresponding beam patterns are plotted

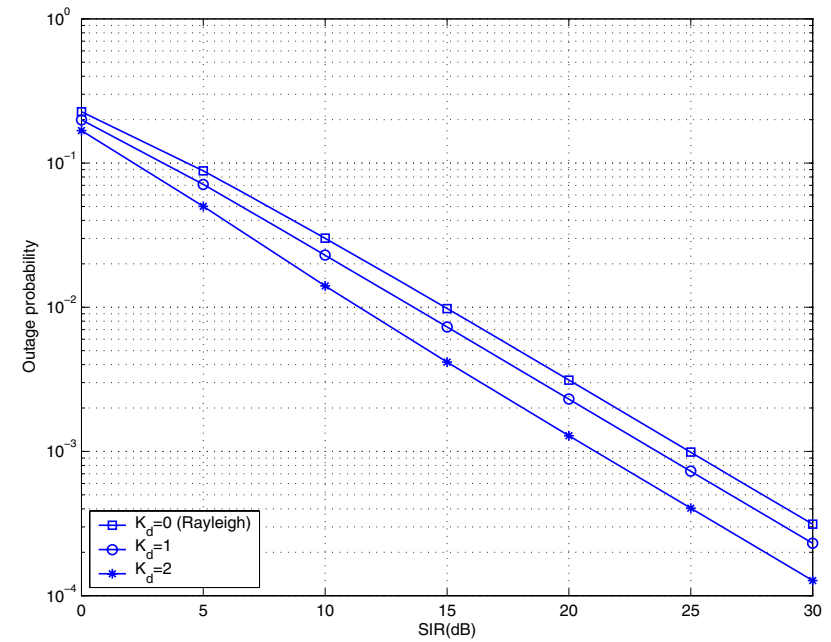

Fig. 5. Outage probability of a wireless system using LCMV beamforming. 3 strongest interferers are at $30^{\circ}, 50^{\circ}$, and $130^{\circ} . N_{I}=7, N=4$, and $M=4$. Desired signal: Rician fading and $90^{\circ}$, interferers: Rayleigh fading.

in Fig. 2. Both desired signal and interferers are subject to Rayleigh fading. At $1 \%$ outage probability, a $14 \mathrm{~dB}$ SIR difference between two interference scenarios is observed in Fig. 4 when LCMV beamforming is considered. This indicates that the outage probability of wireless systems using LCMV beamforming is strongly depends on the directions of the strong interferers. The outage probabilities of conventional beamforming in those two scenarios are also plotted. It is seen that LCMV beamforming has an approximately $2 \mathrm{~dB}$ SIR gain over conventional beamforming when the dominant interferers are at $\left(30^{\circ}, 50^{\circ}, 130^{\circ}\right)$, while the LCMV beamforming suffers a $5 \mathrm{~dB}$ SIR loss when the dominant interferers are at $\left(30^{\circ}, 50^{\circ}\right.$, $95^{\circ}$ ). This illustrates that performance of LCMV beamforming is better than that of conventional beamforming in situations where the dominant interferers are not very close to the desired signal. If an interferer, which is very close to the desired signal, is nulled by LCMV beamforming, the system outage performance can degrade significantly and result in worse performance than that of conventional beamforming. The outage probability results in [10]-[12] which disregard the beam pattern information are also plotted in Fig. 4, and they are unable to model and quantify different interference scenarios and beam patterns.

Fig. 5 and Fig. 6 show the outage probabilities of a wireless system in which the desired signal follows Rician or Nakagami fading. A 4 antenna-element LCMV beamformer is considered. Total 7 interferers and 4 residual interferers are assumed. The DOA's of three strongest interferers are $30^{\circ}$, $50^{\circ}$, and $130^{\circ}$ and the desired signal is at $90^{\circ}$. For two special cases, $K_{d}=0$ in Fig. 5 and $m_{d}=1$ in Fig. 6, the Rician and Nakagami fading cases degenerate into Rayleigh fading and match the Rayleigh fading results plotted in Fig. 4.

\section{CONCLUSIONS}

This paper analyzes the outage performance of wireless systems with LCMV beamforming which cancels a number of dominant interferers. The impact of remaining interferers are examined considering LCMV beam patterns. The fading 


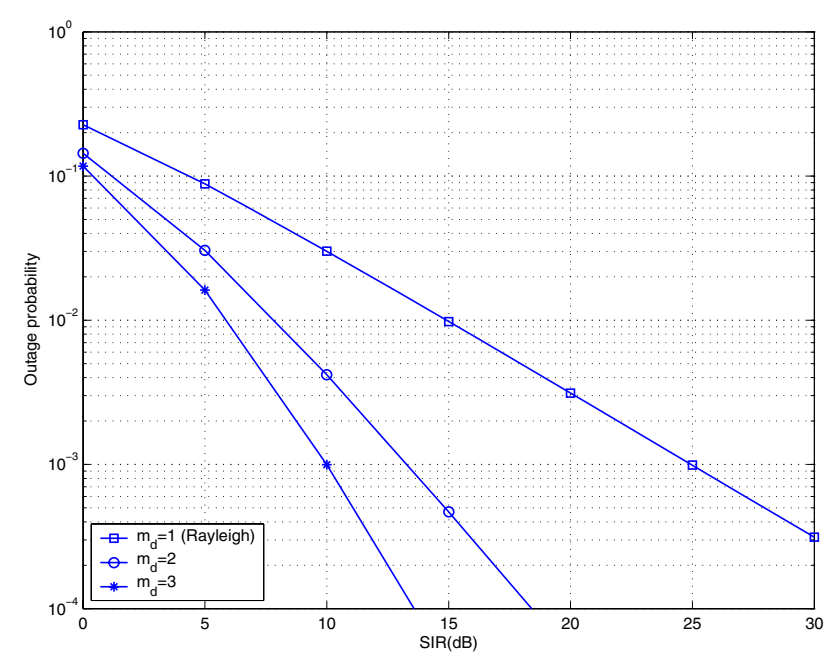

Fig. 6. Outage probability of a wireless system using LCMV beamforming. 3 strongest interferers are at $30^{\circ}, 50^{\circ}$, and $130^{\circ} . N_{I}=7, N=4$, and $M=4$. Desired signal: Nakagami fading and $90^{\circ}$, interferers: Rayleigh fading.

statistics of the desired signal is assumed to be Rayleigh, Rician, or Nakagami, while the interferers are subject to Rayleigh fading. Closed-form outage probability expressions are derived based on a simplified beamforming model. The numerical results show that the outage probability of LCMV beamforming is very sensitive to the directions of the dominant interferers. Unlike [10]-[12], which disregard the directions of dominant interferers and the beam pattern impact on the remaining interferers, the outage performance evaluations presented in this paper fully consider the interference scenarios and LCMV beam patterns. LCMV beamforming and conventional beamforming are also compared in terms of outage performance with considerations of the directions of arrivals of the dominant interferers.

\section{APPENDIX A}

Let $y_{\left(N_{I}\right)} \geq y_{\left(N_{I}-1\right)} \geq \ldots \geq y_{(2)} \geq y_{(1)}>0$ be the order statistics of the interferers. Among them $y_{(N)}, \ldots, y_{(2)}$, and $y_{(1)}$ are $N$ residual interferers in the LCMV beamforming system, and the conditional outage probability for given directions of residual interferers is expressed as, (16),

$$
\begin{aligned}
& P_{\text {out }}\left(\psi_{1}, \psi_{2}, \ldots, \psi_{N}\right) \\
& =\operatorname{Pr}\left[\frac{x}{\sum_{i=1}^{N} G\left(\psi_{i}, \theta, \theta_{1}, \theta_{2}, \ldots, \theta_{M-1}\right) y_{(i)}}<\gamma_{t h}\right] \\
& =\int_{0}^{\gamma_{t h}} \int_{0}^{\infty} y p_{x}(\gamma y) \frac{d}{d y}\left\{\int_{0}^{y} \int_{0}^{\hat{y}_{N-1}} \ldots \int_{0}^{\hat{y}_{1}}\right. \\
& \frac{N_{I} !}{\left(N_{I}-N\right) ! Y^{N}} \exp \left[-\frac{1}{Y}\left(\sum_{n=1}^{N} y_{(n)}+\left(N_{I}-N\right) y_{(N)}\right)\right] \\
& d y_{\left.(1) \ldots d y_{(N-1)} d y_{(N)}\right\} d y d \gamma}
\end{aligned}
$$

It is not straightforward to get a closed-from expression of (A-1) because $y_{(i)}(i=1,2, \ldots, N)$ are not independent. The difficulty can be overcome through introducing new random variables,

$$
\left\{\begin{aligned}
z_{1} & =y_{(1)} \\
z_{i} & =y_{(i)}-y_{(i-1)}
\end{aligned}\right.
$$

The joint pdf of $z_{1}, z_{2}, \ldots, z_{N}$ is found in [10],

$$
\begin{gathered}
p_{z_{1}, z_{2}, \ldots, z_{N}}\left(z_{1}, z_{2}, \ldots, z_{N}\right)=\prod_{i=1}^{N}\left(\frac{N_{I}-i+1}{Y}\right) \\
\times \exp \left(-\frac{z_{i}\left(N_{I}-i+1\right)}{Y}\right)
\end{gathered}
$$

This indicates that the $z_{i}$ 's are independent random variables and the pdf of $z_{i}$ is

$$
p_{z_{i}}\left(z_{i}\right)=\left(\frac{N_{I}-i+1}{Y}\right) \exp \left(-\frac{z_{i}\left(N_{I}-i+1\right)}{Y}\right)
$$

This is an exponential distribution with mean value $Y /\left(N_{I}-i+1\right)$. Now the total interference power $y=$ $\sum_{i=1}^{N} G\left(\psi_{i}\right) y_{(i)}$, which $G\left(\psi_{i}\right)$ is a short notation for $G\left(\psi_{i}, \theta, \theta_{1}, \ldots, \theta_{M-1}\right)$, can be expressed in term of $z_{i}$ as $y=\sum_{i=1}^{N} z_{i} \times\left(\sum_{j=i}^{N} G\left(\psi_{j}\right)\right)$. Let $r_{i}=z_{i} \times \sum_{j=i}^{N} G\left(\psi_{j}\right)$, $i=1,2, \ldots, N$, be new random variables, then $y=\sum_{i=1}^{N} r_{i}$. Due to the independence of $z_{i}$ and notice that $\sum_{j=i}^{N} G\left(\psi_{j}\right)$ are constants, $r_{i}$ 's are independent exponentially distributed random variables with the following pdf

$$
p_{r_{i}}\left(r_{i}\right)=\left(\frac{N_{I}-i+1}{Y \sum_{j=i}^{N} G\left(\psi_{j}\right)}\right) \exp \left(-\frac{\left(N_{I}-i+1\right) r_{i}}{Y \sum_{j=i}^{N} G\left(\psi_{j}\right)}\right)
$$

The MGF of $r_{i}$ can be shown to be

$$
M_{r_{i}}(s)=\left[1-\frac{Y\left(\sum_{j=i}^{N} G\left(\psi_{j}\right)\right)}{N_{I}-i+1} s\right]^{-1}
$$

and the MGF of $y$ can be expressed as a product of MGF of $r_{i}$

$$
M_{y}(s)=\prod_{i=1}^{N} M_{r_{i}}(s)=\prod_{i=1}^{N}\left[1-\frac{Y\left(\sum_{j=i}^{N} G\left(\psi_{j}\right)\right)}{N_{I}-i+1} s\right]^{-1}
$$

Using partial fraction expansions, (A-7) is rewritten as

$$
M_{y}(s)=\sum_{i=1}^{N} A_{i}\left[1-\frac{Y\left(\sum_{j=i}^{N} G\left(\psi_{j}\right)\right)}{N_{I}-i+1} s\right]^{-1}
$$

in which $A_{i}$ is a coefficient,

$$
A_{i}=\prod_{j=1, j \neq i}^{N}\left(1-\frac{C_{i}}{C_{j}}\right)^{-1}
$$

and

$$
C_{i}=\frac{N_{I}-i+1}{\sum_{k=i}^{N} G\left(\psi_{k}\right)}
$$

The above result is valid only if the mean values of $r_{i}$ are mutually different. That is to say,

$$
\operatorname{Pr}\left\{\mathrm{E}\left[r_{m}\right]=\mathrm{E}\left[r_{n}\right]\right\}=0 \text {, for any } m \neq n ; 1 \leq m, n \leq N
$$


This can be confirmed through proof by contradiction. Assuming that (A-11) is not true, we have

$$
\operatorname{Pr}\left\{\mathrm{E}\left[r_{m}\right]=\mathrm{E}\left[r_{n}\right]\right\} \neq 0, \text { for some } m \neq n ; 1 \leq m, n \leq N
$$

then

$$
\operatorname{Pr}\left\{\frac{Y\left(\sum_{j=m}^{N} G\left(\psi_{j}\right)\right)}{N_{I}-m+1}=\frac{Y\left(\sum_{j=n}^{N} G\left(\psi_{j}\right)\right)}{N_{I}-n+1}\right\} \neq 0,
$$

for some $m \neq n ; 1 \leq m, n \leq N$

Without loss of generality, we assume $m>n$, and after some derivation steps, (A-13) can be rewritten as

$$
\operatorname{Pr}\left\{\frac{\sum_{j=m}^{N} G\left(\psi_{j}\right)}{\sum_{j=n}^{m-1} G\left(\psi_{j}\right)}=\frac{(N-m+1)}{(m-n)}\right\} \neq 0,
$$

for some $m \neq n ; 1 \leq m, n \leq N$

Let $q=\frac{\sum_{j=m}^{N} G\left(\psi_{j}\right)}{\sum_{j=n}^{m-1} G\left(\psi_{j}\right)}$. It is seen that $q$ is a continuous random variable due to the fact that $G\left(\psi_{j}\right)$ is a continuous random variable. The probability that a continuous random variable equals a constant value is 0 . Therefore, (A-14) is incorrect and (A-11) is proved by contradiction.

The MGF of total interference $y$ is given by (A-8) and the pdf of $y$ can be derived by taking inverse Laplace transform,

$$
\begin{aligned}
p_{y}(y)= & \sum_{i=1}^{N} A_{i} p_{r_{i}}(y)=\sum_{i=1}^{N} A_{i}\left(\frac{N_{I}-i+1}{Y \sum_{j=i}^{N} G\left(\psi_{j}\right)}\right) \\
& \times \exp \left(-\frac{\left(N_{I}-i+1\right)}{Y \sum_{j=i}^{N} G\left(\psi_{j}\right)} y\right)
\end{aligned}
$$

Let $\gamma=x / y$ denote the SIR. The pdf of the SIR can be found as

$$
p_{\gamma}(\gamma)=\int_{0}^{\infty} y p_{x}(\gamma y) p_{y}(y) d y
$$

Inserting (A-15) into (A-16) we obtain

$$
p_{\gamma}(\gamma)=\sum_{i=1}^{N} A_{i} p_{\gamma \mid r_{i}}(\gamma)
$$

where

$$
p_{\gamma \mid r_{i}}(\gamma)=\int_{0}^{\infty} y p_{x}(\gamma y)\left(\frac{1}{\overline{r_{i}}}\right) \exp \left(-\frac{1}{\overline{r_{i}}} y\right) d y
$$

Notice that $p_{\gamma \mid r_{i}}(\gamma)$ is the pdf of SIR under one Rayleigh faded interferer with mean power $\overline{r_{i}}$ and

$$
\overline{r_{i}}=\frac{Y \sum_{j=i}^{N} G\left(\psi_{j}\right)}{\left(N_{I}-i+1\right)}=\frac{Y}{C_{i}}
$$

Using (A-17), we can write the conditional outage probability as

$$
\begin{gathered}
P_{\text {out }}\left(\psi_{1}, \psi_{2}, \ldots, \psi_{N}\right)=\int_{0}^{\gamma_{t h}} \sum_{i=1}^{N} A_{i} p_{\gamma \mid r_{i}}(\gamma) \\
=\sum_{i=1}^{N} A_{i} \int_{0}^{\gamma_{t h}} p_{\gamma \mid r_{i}}(\gamma)=\sum_{i=1}^{N} A_{i} P_{\text {out } \mid r_{i}}
\end{gathered}
$$

in which $P_{o u t \mid r_{i}}$ is the outage probability under one Rayleigh faded interferer with mean power $\overline{r_{i}}$.

\section{APPENDIX B}

The conditional outage probability using the simplified beamforming model is (Eq. (29)),

$$
\begin{aligned}
& P_{\text {out }}\left(l_{1}, l_{2}, \ldots, l_{N}\right) \\
& =\operatorname{Pr}\left[\frac{x}{\sum_{i=1}^{N}\left(l_{i} y_{(i)}+\left(1-l_{i}\right) \alpha y_{(i)}\right)}<\gamma_{\text {th }}\right] \\
& =\int_{0}^{\gamma_{\text {th }}} \int_{0}^{\infty} y p_{x}(\gamma y) \frac{d}{d y}\left\{\int_{0}^{y} \int_{0}^{\ddot{y}_{N-1}} \ldots \int_{0}^{\ddot{y}_{1}}\right. \\
& \frac{N_{I} !}{\left(N_{I}-N\right) ! Y^{N}} \exp \left[-\frac{1}{Y}\left(\sum_{n=1}^{N} y_{(n)}+\left(N_{I}-N\right) y_{(N)}\right)\right] \\
& \left.d y_{(1)} \ldots d y_{(N-1)} d y_{(N)}\right\} d y d \gamma
\end{aligned}
$$

The total interference power using the simplified beamforming model is

$$
y=\sum_{j=1}^{N}\left(l_{j} y_{(j)}+\left(1-l_{j}\right) \alpha y_{(j)}\right)
$$

where $l_{j}$ is defined by (26). After some derivation steps (B2) can be expressed as $y=\sum_{j=1}^{N} r_{j}$, in which $r_{j}=z_{j} \times$ $\left(\sum_{i=j}^{N}\left(l_{i}+\left(1-l_{i}\right) \alpha\right)\right)$ and $z_{j}$ is defined in (A-2). Similar to the derivations as in Appendix A, it can be shown that $r_{j}$ 's are independently distributed exponential random variables with mean power

$$
\bar{r}_{j}=Y \frac{\sum_{k=j}^{N}\left(l_{k}+\left(1-l_{k}\right) \alpha\right)}{N_{I}-j+1}=\frac{Y}{D_{j}}, j=1,2, \ldots, N
$$

where

$$
D_{j}=\frac{Y}{\bar{r}_{j}}=\frac{N_{I}-j+1}{\sum_{i=j}^{N}\left(l_{i}+\left(1-l_{i}\right) \alpha\right)}, j=1,2, \ldots, N
$$

The MGF of $y$ can be expressed as a product of MGF of $r_{j}$.

$$
M_{y}(s)=\prod_{j=1}^{N} M_{r_{j}}(s)=\prod_{j=1}^{N}\left(1-\bar{r}_{j} s\right)^{-1}=\prod_{j=1}^{J}\left(1-\bar{r}_{(j)} s\right)^{-N_{j}}
$$

Notice that (B-3) represents a vector with $N$ elements, $\overline{\mathbf{r}}_{1}=\left(\bar{r}_{1}\right.$, $\left.\bar{r}_{2}, \ldots, \bar{r}_{N}\right)$. Removing duplicate elements in vector $\overline{\mathbf{r}}_{\mathbf{1}}$, we obtain a new vector with $J$ elements, $\overline{\mathbf{r}}_{(\mathbf{1})}=\left(\bar{r}_{(1)}, \bar{r}_{(2)}, \ldots\right.$, $\left.\bar{r}_{(J)}\right)$. We also have another vector of $J$ elements, $\mathbf{N}=\left(N_{1}\right.$, $N_{2}, \ldots, N_{J}$ ) with each element indicating the number of duplicate elements in the original $\overline{\mathbf{r}}_{1}$ vector. We clearly have $\sum_{j=1}^{J} N_{j}=N$. Similarly, we are able to obtain $D_{(j)}$ from $D_{j}$ (Eq. (B-4)). Using partial fraction expansions, (B-5) can be rewritten as

$$
M_{y}(s)=\sum_{j=1}^{J} \sum_{i=1}^{N_{j}} A_{j i}\left(1-\bar{r}_{(j)} s\right)^{-i}
$$

where $A_{j i}$ is an coefficient due to partial fraction expansions and

$$
A_{j i}=M_{j i} \prod_{k=1 k \neq j}^{J}\left(-\bar{r}_{(k)}\right)^{-N_{k}}, j=1,2, \ldots, J ; i=1,2, \ldots, N_{j}
$$


in which $M_{j i}$ is the residue value corresponding to $1 / \bar{r}_{(j)}$ with order $i$. Notice that (B-6) has a similar form as (A8 ) and, following the derivations from (A-15) to (A-20), the conditional outage probability can be found to be

$$
P_{\text {out }}\left(l_{1}, l_{2}, \ldots, l_{N}\right)=\sum_{j=1}^{J} \sum_{i=1}^{N_{j}} A_{j i} P_{\text {out } \mid r_{(j)}}(i)
$$

where $P_{o u t \mid r_{j}}(i)$ is the outage probability under $i$ independent Rayleigh faded interferers each with mean power $\bar{r}_{(j)}=$ $Y / D_{(j)}$.

\section{REFERENCES}

[1] K. C. Chim, K. C. L. Chan, and R. D. Murch, "Investigating the impact of smart antennas on SAR," IEEE Trans. Antennas Propagat., vol. 52, no. 5, pp. 1370-1374, May 2004.

[2] R. B. Ertel, P. Cardieri, K. W. Sowerby, T. S. Rappaport, and J. H. Reed, "Overview of spatial channel models for antenna array communication systems," IEEE Pers. Commun. Mag., vol. 5, no. 1, pp. 10-22, Feb. 1998.

[3] V. Tarokh, N. Seshadri, and A. R. Calderbank, "Space-time codes for high data rate wireless communication: performance criterion and code construction," IEEE Trans. Inform. Theory, vol. 44, no. 2, pp. 744-765, Mar. 1998.

[4] H. L. V. Trees, Optimum Array Processing, Part IV of Detection, Estimation, and Modulation Theory. New York: Wiley Interscience, 2002.

[5] J. H. Winters, "Smart antennas for wireless systems," IEEE Pers. Commun. Mag., vol. 5, no. 1, pp. 23-27, Feb. 1998.

[6] H. J. Li and T. Y. Liu, "Comparison of beamforming techniques for WCDMA communication systems," IEEE Trans. Veh. Technol., vol. 52, no. 4, pp. 752-760, July 2003.

[7] F. A. C. M. Cardoso, M. A. C. Fernandes, and D. S. Arantes, "Spacetime processing for smart antennas in advanced receivers for the user terminal in 3G WCDMA systems," IEEE Trans. Consumer Electron., vol. 48, no. 4, pp. 1082-1090, Nov. 2002.

[8] M. Fan and E. Esteves, "The impact of antenna-array receivers on the reverse link performance of CDMA $20001 \mathrm{xEV}$ high rate packet data systems," in Proc. IEEE 58th VTC 2003-Fall, Oct. 2003, vol. 2, pp. 6-9.

[9] J. H. Reed, Software Radio: A Modern Approach to Radio Engineering, 1st ed. Upper Saddle River, NJ: Prentice Hall, 2002.

[10] M. Hasna, M.-S. Alouini, A. Bastami, and E. S. Ebbini, "Performance analysis of cellular mobile systems with successive co-channel interference cancellation," IEEE Trans. Wireless Commun., vol. 2, no. 1, pp. 29-40, Jan. 2003.

[11] M. Kang, M.-S. Alouini, and L. Yang, "Outage probability and spectrum efficiency of cellular mobile radio systems with smart antennas," IEEE Trans. Commun., vol. 50, no. 12, pp. 1871-1877, Dec. 2002.

[12] R. Mostafa, A. Annamalai, and J. H. Reed, "Performance evaluation of cellular mobile radio systems with interference nulling of dominant interferers," IEEE Trans. Commun., vol. 52, no. 2, pp. 326-335, Feb. 2004.
[13] A. Abu-Dayya and N. C. Beaulieu, "Outage probabilities of cellular mobile systems with multiple Nakagami interferers," IEEE Trans. Veh. Technol., vol. 40, no. 6, pp. 757-768, Nov. 1991.

[14] G. K. Karagiannidis, S. A. Kotsopoulos, and P. T. Mathiopoulos, "Generalized approach for the evaluation of outage performance in micro- and pico-cellular networks," IEE Proc. Commun., vol. 149, no. 2, pp. 123-128, Apr. 2002.

[15] G. K. Karagiannidis, "Performance analysis of SIR-based dual selection over correlated Nakagami- $m$ fading channels," IEEE Trans. Veh. Technol., vol. 52, no. 5, pp. 1207-1216, Sep. 2003.

[16] V. Emamian, M. Kaveh, and M.-S. Alouini, "Outage probability with transmit and receive diversity in a shadowing environment," in Proc. IEEE WCNC, Mar. 2002, vol. 1, pp. 17-21.

[17] C. Chayawan and V. A. Aalo, "On the outage probability of optimum combining and maximal ratio combining schemes in an interferencelimited Rice fading channel," IEEE Trans. Commun., vol. 50, no. 4, pp. 532-535, Apr. 2002.

[18] M. K. Simon and M.-S. Alouini, Digital Communication Over Generalized Fading Channels: A Unified Approach to the Performance Analysis. New York: John Wiley \& Sons, 2000.

[19] H. Li, Y. D. Yao, and J. Yu, "Outage probabilities of wireless systems with imperfect beamforming," IEEE Trans. Veh. Technol., to be published.

[20] J. Yu and Y.-D. Yao, "Reverse link capacity of SIR-based power controlled CDMA systems with antenna arrays," J. Wireless Commun. Mobile Comput., vol. 3, no. 6, pp. 293-297, Sep. 2003.

[21] J. C. Liberti and T. S. Rappaport, Smart Antennas for Wireless Communications: IS-95 and Third Generation CDMA Applications. Upper Saddle River, NJ: Prentice Hall, 1999.

[22] F. Rashid-Farrokhi, K. J. R. Liu, and L. Tassiulas, "Transmit beamforming and power control for cellular wireless systems," IEEE J. Select. Areas Commun., vol. 16, no. 8, pp. 1437-1450, Oct. 1998.

[23] S. Haykin, Adaptive Filter Theory, 3rd ed. Upper Saddle River, NJ: Prentice Hall, 1986.

[24] Y.-D. Yao and A. U. H. Sheikh, "Investigations into cochannel interference in microcellular mobile radio systems," IEEE Trans. Veh. Technol., vol. 41, no. 2, pp. 114-123, May 1992.

[25] _ - "Outage probability analysis for microcell mobile radio systems with cochannel interferers in Rician/Rayleigh fading environment," IEEE Electron. Lett., vol. 26, no. 13, pp. 864-866, June 1990.

[26] M. Abramowitz and I. A. Stegun, Handbook of Mathematical Functions with Formulas, Graphs, and Mathematical Tables, 9th ed. New York: Dover, 1970.

[27] A. F. Naguib, A. Paulraj, and T. Kailath, "Capacity improvement with base-station antenna arrays in cellular CDMA," IEEE J. Select. Areas Commun., vol. 43, no. 3, pp. 691-698, Aug. 1994.

[28] U. Spagnolini, "A simplified model for probability of error in DS CDMA systems with adaptive antenna arrays," in Proc. IEEE Int. Conf. Commun., June 2001, vol. 7, pp. 2271-2275.

[29] J. Yu, Y. D. Yao, A. F. Molisch, and J. Zhang, "Performance evaluation of CDMA reverse links with imperfect beamforming in a multicell environment using a simplified beamforming model," IEEE Trans. Veh. Technol., vol. 55, no. 3, pp. 1019-1031, May 2006. 